\title{
Early stages of the HFCVD process on multi-vicinal silicon surfaces studied by electron microscopy probes ( SEM, TEM)
}

\author{
J.C. Arnault ${ }^{\mathrm{a}, *}$, S. Pecoraro ${ }^{\mathrm{a}}$, J. Werckmann ${ }^{\mathrm{a}}$, F. Le Normand ${ }^{\mathrm{a}}$, N. Motta ${ }^{\mathrm{b}}$, \\ R. Polini ${ }^{\mathrm{b}}$ \\ ${ }^{a}$ Groupe Surfaces-Interfaces, Institut de Physique et Chimie de Strasbourg, IPCMS-GSI, UMR 7504, Bat 69, 23, rue du Loess, 67037 \\ Strasbourg, France \\ ${ }^{\mathrm{b}}$ Physics Department, Universita Tor Vergata, Via della Ricerca Scientifica, 00133 Rome, Italy
}

\begin{abstract}
In this paper, we show that silicon dimples are suitable samples to study diamond nucleation on a controlled distribution of defects by SEM FEG and HRTEM observations. Indeed, multi-vicinal surfaces generated by a UHV thermal treatment have been characterised by STM experiments. On these terraces, we observed a strong increase of the nucleation density higher than two orders of magnitude compared to pristine silicon samples. Moreover, a preferential location of diamond nuclei along the steps is reported. This result is explained by the large surface diffusion length of carbon species compared to the terrace's width. Indeed, during the early stages of growth, oriented silicon carbide nano-crystals are observed with the relationship $\mathrm{SiC}(220) / / \mathrm{Si}(220)$. ( 2001 Elsevier Science B.V. All rights reserved.
\end{abstract}

Keywords: Diamond; Nucleation; Silicon step; Transmission electron microscopy

\section{Introduction}

Diamond nucleation and growth on silicon surfaces has been extensively studied [1]. Today, the introduction of highly textured diamond films in microelectronic devices working at high power and high temperature remains a major challenge [2]. Therefore, a deeper knowledge of the elemental mechanisms is needed to control diamond growth. Indeed, the early stages of diamond nucleation are poorly understood. The chemical nature and the structure of the carbon phase precursor of the diamond still remain unclear. They strongly depend on the CVD experimental conditions. Recently, local direct epitaxy of diamond on a silicon step was reported for HFCVD with a bias enhanced

\footnotetext{
${ }^{*}$ Corresponding author. Tel.: +33-3-88-10-7102; fax: +33-3-8810-7248.

E-mail address: jean-charles.arnault@ipcms.u-strasbg.fr (J.C. Arnault).
}

nucleation (BEN) step [3,4]. Another TEM study emphasised the major part of redeposited silicon islands on to which silicon carbide nanocrystals grew before diamond nucleation by MWCVD with a BEN step [5]. Moreover, on silicon, previous studies have emphasised the effect of pre-existent structural defects which provide more or less preferential sites for nucleation: apex of pyramids [6], sharp edges [7], and scratching grooves $[8,9]$. On clean silicon surfaces, Polini [10] showed that steps act as preferential sites for diamond nuclei.

We report here on diamond nucleation obtained on multi-vicinal surfaces generated by thermal UHV treatment. We show that such samples are suitable to obtain a controlled distribution of structural defects. Starting with clean monocrystalline samples is a major requirement to better understand the fundamental processes of nucleation. In this way, neither substrate pre-treatment nor bias step were used. On terraces and steps created by a thermal flash, there was strong enhancement of diamond nucleation density, higher 
than two orders of magnitude. The edges of these defects act as nucleation preferential sites. Silicon carbide nanocrystals oriented according to the relationship $\operatorname{SiC}(220) / / \operatorname{Si}(220)$ were observed at the early stages of diamond growth, by high resolution TEM on the top view areas.

\section{Experimental}

The experimental procedure to prepare silicon dimples was previously reported [11,12]. Si discs of 3-mm diameter were cut from phosphorus-doped $\mathrm{Si}(111)$ wafers of resistivity $1-3 \Omega \cdot \mathrm{cm}$. A mechanical polishing of the two faces was performed and the final thickness of the sample was $150 \mu \mathrm{m}$. One face was polished by abrasion up to $0.5 \mu \mathrm{m}$ and underwent a final polishing by a colloidal solution leading to a clean surface suitable for top view observations by high resolution TEM. The polishing of the second face was limited to $3 \mu \mathrm{m}$. A dimple was ground on this face, using a GATAN dimpler, the depth of the dimple and its diameter were $100 \mu \mathrm{m}$ and $2.4 \mathrm{~mm}$, respectively.

Some samples were further thinned down at the bottom of the dimple by bombardment with $\mathrm{Ar}^{+}$ions accelerated at $6 \mathrm{kV}$ with an incidence angle of $15^{\circ}$. The amorphisation of the bombarded area was avoided by cooling the sample at liquid nitrogen temperature.

The samples were transferred in an analysis chamber where a UHV cleaning procedure was performed: heating by electronic bombardment with steps at $1123 \mathrm{~K}$ during $15 \mathrm{~min}$ followed by a flash up to $1573 \mathrm{~K}$. The treatment efficiency was checked by STM experiments performed with a UHV OMICRON microscope, directly connected to the preparation chamber where thermal treatments were performed. STM observations showed the formation of multi-vicinal surfaces. In Fig. $1 \mathrm{a}$, the terraces have a mean width of $6 \mathrm{~nm}$. Reconstructions $(7 \times 7)$ observed on these terraces show proof of the sample cleanliness (Fig. 1b).

Then, a HFCVD synthesis was performed in our reactor directly connected to the analysis chamber. The main deposition conditions are listed below: $\mathrm{CH}_{4} / \mathrm{H}_{2}$ content, $0.5 \%$; pressure, $30 \mathrm{mbar}$; flow rate, $200 \mathrm{sccm}$; tungsten filaments temperature, $2373 \mathrm{~K}$; sample temperature, $1073 \mathrm{~K}$.

SEM observations were first performed on a JEOL JSM 840 microscope operating at $10 \mathrm{kV}$ yielding a lateral resolution of $20 \mathrm{~nm}$. Then, higher resolution was reached during the SEM FEG observations on a PHILIPS XL 30 microscope working at $5 \mathrm{kV}$.

High-resolution TEM observations were performed on a TOPCON $002 \mathrm{~B}$ working at $200 \mathrm{keV}$ with a pointto-point resolution of $0.18 \mathrm{~nm}$.
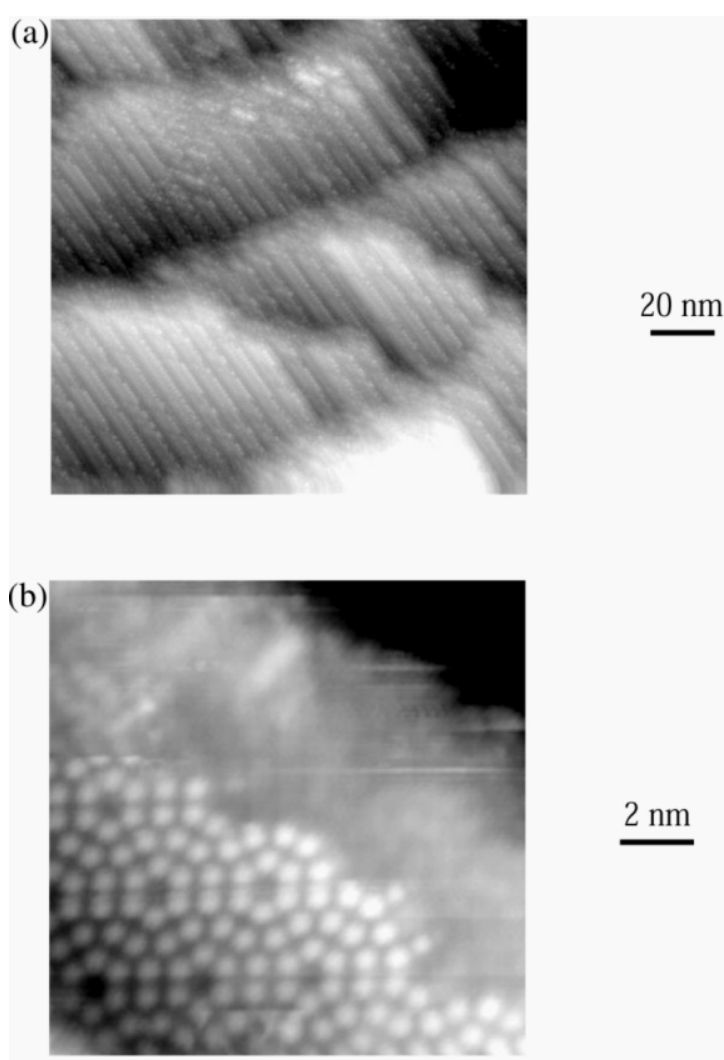

Fig. 1. (a) STM image $(150 \mathrm{~nm} \times 150 \mathrm{~nm})$ of terraces induced by the UHV thermal treatment. The width of the terraces is $6 \mathrm{~nm}$. The $Z$ scale of these images is between 0 and $1.8 \mathrm{~nm}$. (b) STM image (13.3 $\mathrm{nm} \times 13.3 \mathrm{~nm})$ of a $(7 \times 7)$ reconstructed terrace. The $Z$ scale of these images is between 0 and $0.51 \mathrm{~nm}$.

\section{Results}

\subsection{Dimple's borders: SEM observations}

SEM observations on the borders of the dimple where stepped surfaces were obtained led to three main conclusions:

1. the nucleation density increased strongly compared to pristine silicon samples;

2. diamond nuclei are located preferentially along steps;

3. small islands coexisting with diamond crystals were observed.

Diamond nucleation densities calculated from SEM images are shown in Table 1 . The nucleation density depends strongly on the orientation of the terraces into the dimple. After $15 \mathrm{~min}$ of deposition, the density value reached $2 \times 10^{8}$ islands $\mathrm{cm}^{-2}$ on the denser terraces (Fig. 2a). This value is 400 times higher than the diamond density on the pristine $\mathrm{Si}(111)$ [13]. A linear density could be calculated along the edges. It is included in the range $1-1.5 \times 10^{4}$ nuclei $\mathrm{cm}^{-1}$. After 
Table 1

Comparison of diamond nucleation densities vs. deposition time between $\mathrm{Si}(111)$ dimples and pristine $\mathrm{Si}(111)$ samples

\begin{tabular}{lllll}
\hline & $15 \mathrm{~min}$ & $30 \mathrm{~min}$ & $180 \mathrm{~min}$ & $240 \mathrm{~min}$ \\
\hline Si(111) dimple & $2 \times 10^{8} \mathrm{~cm}^{-2}$ & $2 \times 10^{8} \mathrm{~cm}^{-2}$ & $1.2 \times 10^{8} \mathrm{~cm}^{-2}$ & - \\
Pristine Si(111) & $5 \times 10^{5} \mathrm{~cm}^{-2}$ & $2 \times 10^{6} \mathrm{~cm}^{-2}$ & $8 \times 10^{6} \mathrm{~cm}^{-2}$ & $10^{7} \mathrm{~cm}^{-2}$ \\
\hline
\end{tabular}

$180 \mathrm{~min}$, the diamond nucleation density reaches a saturation value due to the coalescence of diamond islands (Fig. 2b).

During diamond deposition, atomic hydrogen coming from the plasma interacts with the silicon surface and this leads to a preferential etching along the (100) planes as shown by our previous AFM study [14]. This result is supported by the higher stability of the $\mathrm{SiH}_{2}$ precursor on these planes $[15,16]$. In addition to the terraces and steps, such etch features are observed (Fig. 2a). The SEM images show that the diamond nucleation is heterogeneous, nuclei are located preferentially along the steps and at the edges of etch pits.

In addition, small islands with a diameter of several tens of nanometers are coexisting with diamond crystals. After $180 \mathrm{~min}$ of deposition, their density is $8 \times 10^{9}$

\section{(a)}

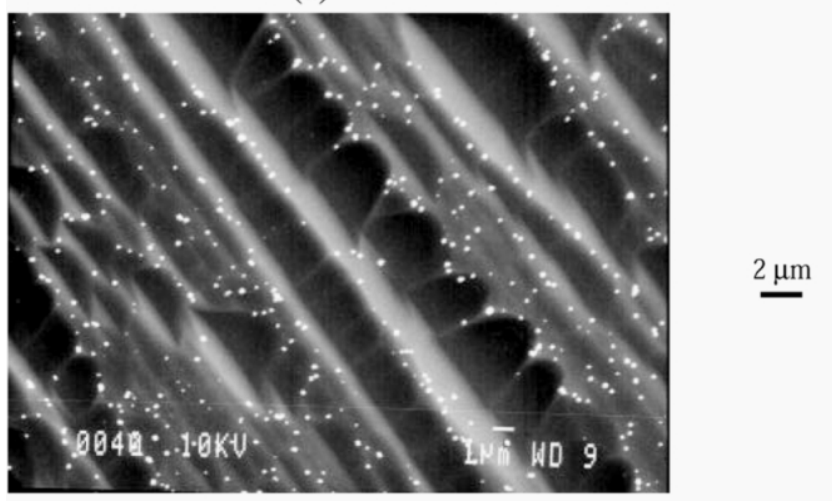

(b)

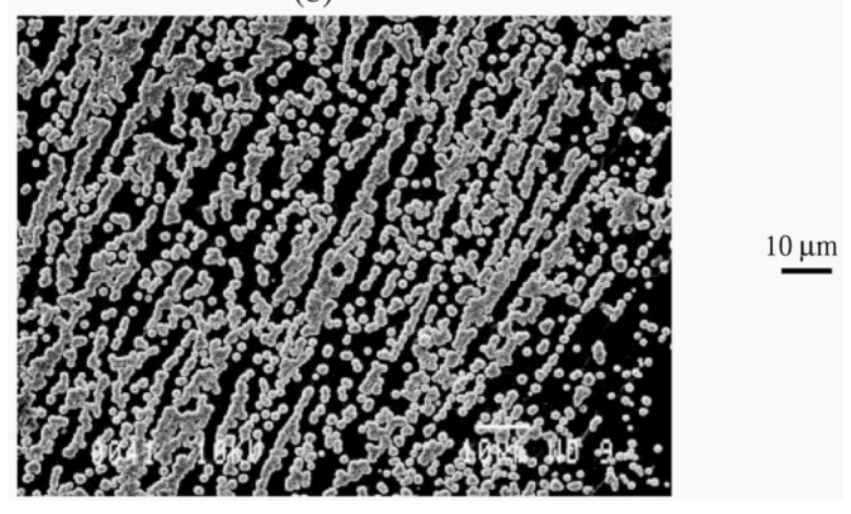

Fig. 2. (a) SEM image of a silicon dimple after $30 \mathrm{~min}$ of diamond deposition showing the preferential location of the nuclei. (b) SEM image of a silicon dimple after $180 \mathrm{~min}$ of diamond deposition. $\mathrm{cm}^{-2}$. Some of these islands are facetted (Fig. 3) and could constitute diamond precursors. The reason why they do not grow is not yet clear. It is probably related to the chemical nature of these islands which is still unknown. The double distribution was already observed in our study of diamond growth on pristine silicon flat samples [17]. HRTEM observations of cross-sections are in progress.

\subsection{Thinned silicon areas: HRTEM observations}

To study the early stages of growth, a HFCVD synthesis of $7 \mathrm{~min}$ was performed on thinned silicon crystalline areas. Two main zones of different thickness are observable.

A SAD pattern realised on the thinner silicon area is shown in Fig. 4. The detailed exploitation is given in Table 2. In addition to the diffraction spots noted, a, b, c, corresponding to the diffraction by (110), (220) and (422) silicon planes, rings labelled 1, 2, 3 correspond to d-spacings of $0.252,0.154$ and $0.131 \mathrm{~nm}$, in good agreement with $\beta$-SiC (111), (220) and (311) values, respectively. The strong texture of the (220) silicon carbide ring suggests a preferential orientation of the carbide crystals according to the relation $\mathrm{SiC}(220) / / \mathrm{Si}(220)$. However, these nano-crystals are disoriented to each other leading to rings into the diffraction pattern. Moreover, dark field observations done with $\beta-\mathrm{SiC}$ (220) spots reveal a distribution of nano-crystals with a size included between 10 and $20 \mathrm{~nm}$.

Bright field images of nano-crystals reveal $\beta$-SiC crystals. The measured d-spacings are $0.213 \pm 0.008 \mathrm{~nm}$ and $0.248 \pm 0.006 \mathrm{~nm}$ in good agreement with the $d_{\text {hkl }}$ of $\beta$-SiC (200) and (111) planes, respectively (Fig. 5). The bright field image of the second nano-crystal (Fig. 5 b) which reveals (200) $\beta$-SiC planes, shows facets indicated by white arrows.

On the second observable silicon area, HRTEM experiments showed the presence of etching features with threefold symmetry [14]. Dark field images done with $\beta$-SiC (220) spots showed that the silicon carbide is mainly located inside the features. This result supports previous scanning auger microscopy observations [13].

\section{Discussion}

Let us discuss this significant enhancement of dia- 

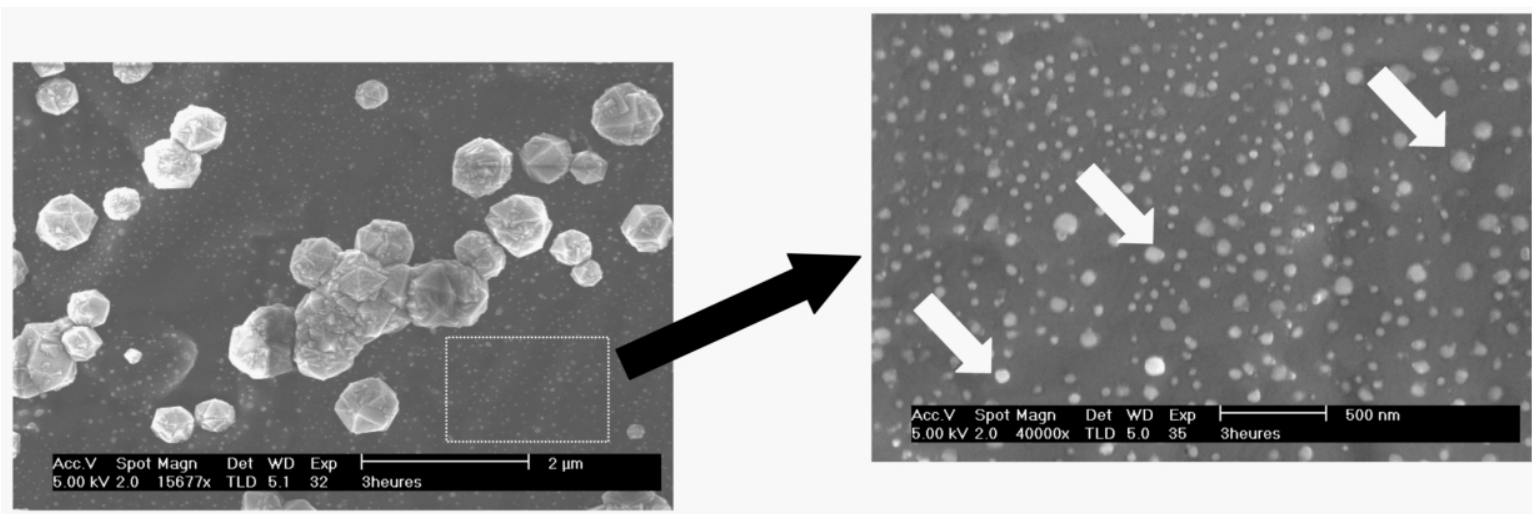

Fig. 3. FEG SEM image of a silicon dimple after $180 \mathrm{~min}$ of diamond deposition showing small islands coexisting with diamond crystals. In the enlarged area, some facetted islands are indicated by arrows.

Table 2

Complete exploitation of SAD pattern realised on thinned silicon areas

\begin{tabular}{lllllll}
\hline & $\mathrm{Si}$ & & & $\beta-\mathrm{SiC}$ & \\
& $\mathrm{D}_{\mathrm{hkl}}(\AA)$ & $(\mathrm{hkl})$ & & $\mathrm{D}_{\mathrm{hkl}}(\AA)$ & $(\mathrm{hkl})$ & \\
\hline $\mathrm{a}$ & 3.84 & $(110)$ & & 2.52 & $(111)$ & 1 \\
$\mathrm{~b}$ & 1.92 & $(220)$ & & 1.54 & $(220)$ & 2 \\
$\mathrm{c}$ & 1.10 & $(422)$ & & 1.31 & $(311)$ & 3 \\
\hline
\end{tabular}

mond nucleation on vicinal surfaces. On a stepped surface, there is a clear preferential nucleation on the vicinity of steps. This observation is in close relation with the high energy of such sites. Indeed, the number of dangling bonds is higher in the vicinity of these sharp edges $[18,19]$ so that the carbon saturation is more quickly reached $[20,21]$. However, at the present time, the SEM observations did not allow us to locate diamond nuclei at the top of the steps. STM experiments are in progress to identify the nucleation site, either at the top or at the bottom of a step.

The nucleation process is governed by two main

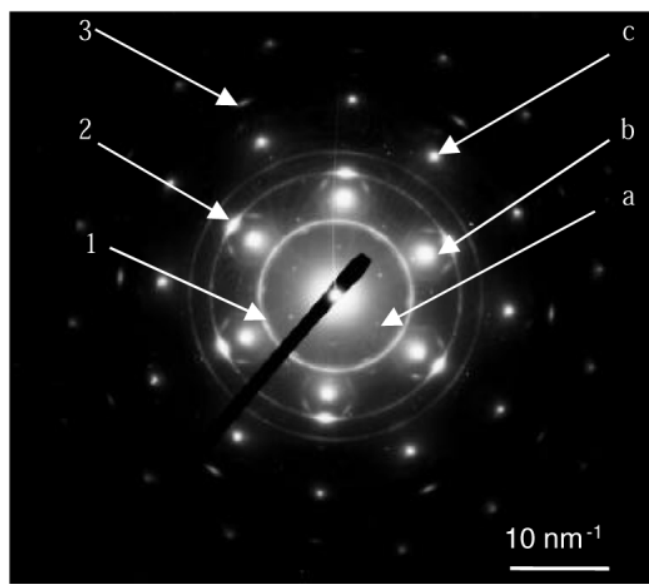

Fig. 4. SAD pattern realised on a thinned silicon area. The complete exploitation is gathered in Table 2. parameters: the density of nucleation sites $N$ and the surface diffusion length $\lambda_{\mathrm{N}}$ defined as the mean path
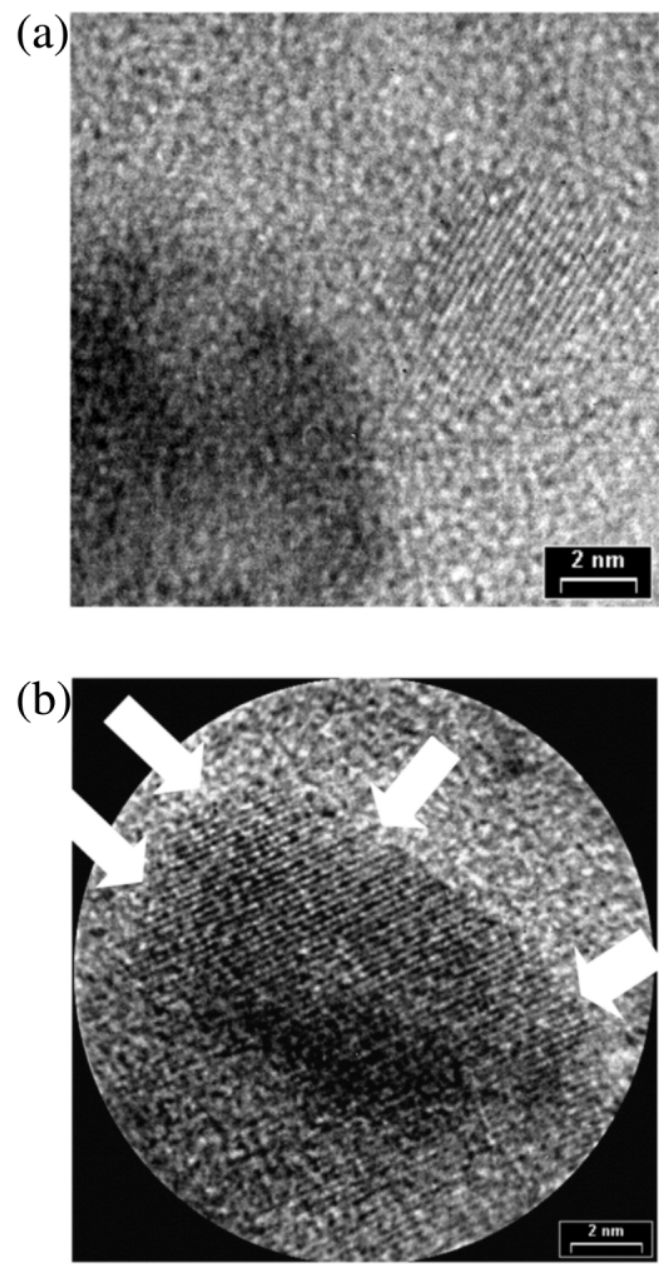

Fig. 5. (a) Bright field image of a $\beta$-SiC nano-crystal. The d-spacing is $0.248 \mathrm{~nm}$, in good agreement with the one from $\beta$-SiC (111) (cf. Table 2). (b) Bright field image of another $\beta$-SiC nano-crystal. In this case, the d-spacing is $0.213 \mathrm{~nm}$, in good agreement with the one from $\beta$-SiC (200) which is $0.218 \mathrm{~nm}$. This nano-crystal exhibits facets indicated by white arrows. 
of the carbon precursor before reacting or desorbing. The effect of the surface diffusion will be efficient only if $\lambda_{\mathrm{N}}$ is larger than the mean distance between two terraces named $D$. We assume here that the surface diffusion of the carbon precursor is low relative to the desorption or competitive reaction such as carbide formation. Starting from a similar description for the initial growth of the diamond nuclei, we estimated the mean diffusion path of the carbon precursor to $0.1-0.15$ $\mu \mathrm{m}$ [22]. As shown by STM images (Fig. 1), the width of the terraces is of several nanometers so that $\lambda_{N}$ is much larger than $D$ and the probability for diffusing carbon species to reach the edge of a terrace is high.

Top view high-resolution TEM observations of the thinned area show the formation of oriented $\beta$-SiC nano-crystals during the early stages. It is tempting to suppose that these crystals should be the smaller islands observed on the vicinal surfaces, coexisting with diamond crystals. This idea is supported by previous studies [5,23]. Further diamond depositions are in progress to observe the carbon phase which constitutes the diamond precursor.

\section{Conclusion}

We show that silicon dimples are suitable samples to obtain a distribution of controlled defects. On these samples, a study of the early stages of diamond nucleation is possible. First the top view TEM observations of thinned down silicon area showed the formation of nano-crystals of $\beta-\mathrm{SiC}$ during the early stages of diamond growth. These crystals are preferentially oriented along (220) directions. For a deposition time of $15 \mathrm{~min}$, the diamond nucleation on the borders of the silicon dimple is strongly enhanced on the terraces and the nuclei are located along the steps. SEM observations showed the presence of small islands coexisting with diamond crystals. However, several opening questions remain and further investigations are now in progress. Sequential CVD deposits being performed on the thinned down silicon areas to determine the diamond precursor nature and structure. EELS experiments are planned to study the chemical nature of the precursors. These studies will be completed with cross-sections of dimples to analyse the interfaces silicon-silicon carbide-diamond and to determine the epitaxial relationship. Finally, STM UHV experiments on samples which underwent short diamond deposition are also in progress to correlate the location of the diamond nuclei with the defect distribution. At the present time, the SEM FEG observations suggest that the nucleation sites are located in the vicinity of the steps, STM experiments should be valuable to identify with an atomic resolution the nuclei positions vs. a step, either at the top or at the bottom.

\section{Acknowledgements}

Authors are grateful to A. Redjaimia and J. Poisson from the Ecole des Mines of Nancy for giving access to a SEM FEG microscope. We also thank G. Wurtz who performed ion bombardment to prepare thin samples for HRTEM.

\section{References}

[1] H. Liu, D.S. Dandy, Diamond Chemical Vapor Deposition, Noyes publications, Park Ridge, New Jersey, 1995.

[2] D.M. Gruen, I. Buckley-Golder, Diamond Films: recent developments, MRS Bulletin, 1998.

[3] X. Jiang, C.L. Jia, Phys. Rev. Lett. 84 (2000) 3658.

[4] S.T. Lee, H.Y. Peng, X.T. Zhou et al., Science 287 (2000) 104.

[5] W. Jäger, D. Wittorf, C.L. Jia, U. Urban, in: P. Vincenzini (Ed.), Proceedings 9th CIMTEC, 1998.

[6] R. Ramesham, C. Ellis, J. Mater. Res. 7 (1992) 1189.

[7] P.A. Dennig, D.A. Stevenson, Appl. Phys. Lett. 59 (1991) 1562.

[8] L. Demuynck, J.C. Arnault, C. Speisser, R. Polini, F. Le Normand, Diam. Relat. Mater. 6 (1997) 235.

[9] S. Yugo, A. Izumi, T. Kanai, T. Muto, T. Kimura, 2nd Int. Conf. on New Diamond Science and Technology, Washington, D.C., 1991.

[10] R. Polini, J. Appl. Phys. 72 (1992) 2517.

[11] I. Vianey, Thesis, University of Marseille, France, 1994.

[12] M. Hanbücken, I. Vianey, A. Degiovanni et al., Appl. Surf. Sci. 72 (1993) 79.

[13] L. Demuynck, Ph.D. Thesis, University of Strasbourg, France, December 1995.

[14] J.C. Arnault, S. Hubert, F. Le Normand, J. Phys. Chem. B 312 (1998) 4856.

[15] J. Boland, J. Surf. Sci. 261 (1992) 17.

[16] Y. Wei, L. Lian, I.S.T. Tsong, Appl. Phys. Lett. 66 (1995) 1818.

[17] J.C. Arnault, S. Hubert, L. Demuynck et al. (to be submitted).

[18] K. Kobayashi, N. Mutsumura, Y. Machi, Mater. Manuf. Proc. 7 (1992) 395.

[19] Ligenza, Phys. Chem. 65 (1961) 2011.

[20] B. Lux, R. Haubner, in: R.E. Clausing, L.L. Horton, J.C. Angus, P. Koidl (Eds.), Diamond and Diamond-like Films and Coatings, Plenum Press, New York, 1991.

[21] J.C. Angus, Y. Wang, M. Sunkara, Ann. Rev. Mater. Sci. 21 (1991) 221.

[22] F. Le Normand, J.C. Arnault, L. Fayette, B. Marcus, M. Mermoux, V. Parasote, J. Appl. Phys. 80 (1996) 1830.

[23] S. Yugo, T. Kimura, H. Kanai, in: S. Saito, O. Fukunaga, M. Yoshikawa (Eds.), Science and Technology of New Diamond, Tokyo, , 1990, p. 119. 